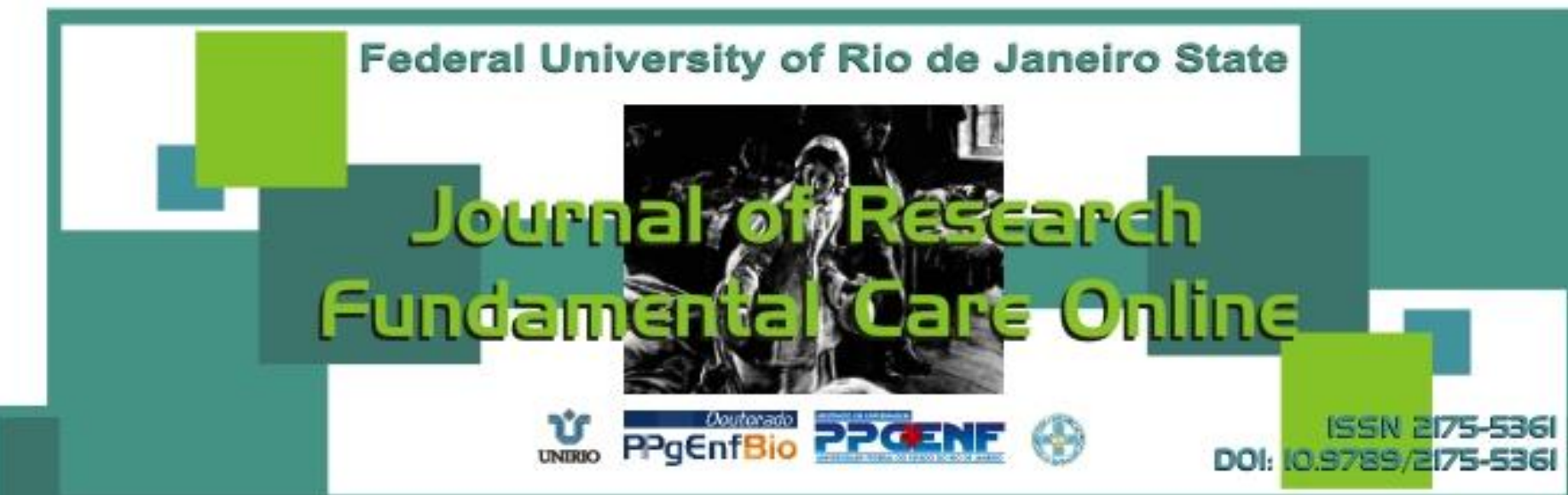

EDITORIAL

\title{
Neurotrauma Research in Latin America
}

Andrés M. Rubiano ${ }^{1}$, Angela $M$. Rios $^{2}$

Recently, the global burden of disease study, presented by the world health organization, call the attention of the research community, showing that trauma still remains as a major public health problem in Central and South America. 1

Within the large spectrum of this problem, one of the most specific injuries associated with disability and death is head trauma. Unfortunately, research groups evaluating integral aspects of the management of this disease and seeking to reduce their impact on society are scarce in this region of the world. 2

Brazil, Colombia, Argentina and Cuba are among the few countries that are engaged in research in neurotrauma.

Few basic and clinical studies have been performed multicentric in our region and most of the used evidence in the comprehensive management of this disease, comes from studies conducted in Europe and North America. In these regions, care systems differ in key aspects such available resources for advanced neuromonitoring at intensive care units.

Recently a study performed in Ecuador, Argentina and Bolivia, published in the New England Journal of Medicine, generated international controversy due to the implications of their results in the treatment protocols of severe head trauma. 3

After deep analysis of the results of this study in several meetings of traumatic brain injury experts and after extensive academic discussions published in different biomedical journals, it was considered that this study, with a very good methodological approach, failed trying to understand the ecology of the medical management in environments with slightly different views of advanced care.4-8

These patients were managed under non traditional high income health care settings; due to this, interpretation of advanced monitoring to generate intervention decisions, could be influenced by the idiosyncrasies and pre-existing conditions in different health systems and training resources.

This interesting experience, coming from this trial, should provide an opportunity for neurotrauma research groups in Latin America (where large volumes of patients are generated by our social conflicts), closing a gap, where ecology of patient care inside different systems of each region (which may even have variation within the same state), need to be taken into account in further clinical studies. 
These elements are fundamental in the process of identifying issues related to human factor and their interaction with new or old medical technology, including analysis of the training process and the variability that these aspects can induced in the research results, either in terms of outcome or mortality.

It is time to think about how consortium studies related to neurotrauma in Latin America are needed, in order to integrate robust databases that reveal the true variability in the comprehensive care of these patients, including aspects of pre-hospital care, emergency care, surgical and intensive care management, without neglecting fundamental aspects like integral rehabilitation.

It is time to develop a Latin American traumatic coma data bank. This initiative should be led by research groups from the region, trying to understand and integrate data analysis in a better fashion, trying to reduce the potential of bias as a result of misunderstanding the dynamic of health systems with limited resources, low capabilities for urgent inter-hospital referral and lack subspecialty training that is still in the process of development.

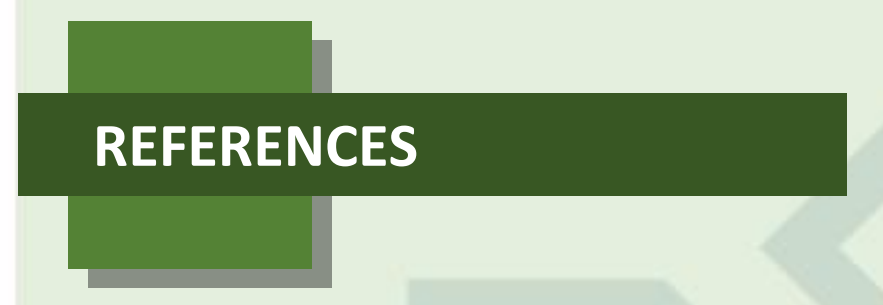

1. Byass P, de Court, Graham WJ, Laflamme L, McCaw-Binns A, Sankoh OA, et al. Reflections on the global burden of disease 2010 estimates. PLoS Med 2013; 10(7): e1001477.

2. Puvanachandra P, Hyder A. Traumatic brain injury in Latin America and the Caribbean: a call for research. Salud pública Méx; 2008, 50 (1) pp. s3-s5.

3. Chesnut RM, Temkin N, Carney N, Dikmen S, Rondina C, Videtta W, Petroni G, Lujan S, Pridgeon J, Barber J, Machamer J, Chaddock K, Celix J, Cherner M, Hendrix T, for the Global Neurotrauma Research Group. A trial of intracranial-pressure monitoring in traumatic brain injury. N Engl J Med 2012;367:2471-81.

4. Sahuquillo J, Biestro A. Is intracranial pressure monitoring still required in the management of severe traumatic brain injury? ethical and methodological considerations on conducting clinical research in poor and low-income countries. Surg Neurol Int. 2014; 5: 86.

5. Murillo-Cabezas F, Godoy DA. Intracranial pressure monitoring in severe traumatic brain injury: A different perspective of the BestTrip trial. Med Intensiva. 2014; 38: 237-9.

6. Rubiano AM, Puyana JC. Intracranial pressure monitoring in traumatic brain injury. N Engl J Med. 2013; 368(18):1748.

7. Le Roux P. Intracranial pressure after the best trip trial: a call for more monitoring. Curr Opin Crit Care. 2014; 20(2):141-7.

8. Chesnut RM. Intracranial pressure monitoring: headstone or a new head start. The BEST TRIP trial in perspective. Intensive Care Med. 2013; 39(4):771-4.

${ }^{1}$ Professor of Neurosciences, South Colombian University, Neiva (Colombia), Director of Neurotrauma Research, El Bosque University, Bogotá (Colombia), Medical and Research Director, MEDITECH Foundation, Neiva (Colombia), Chair of Neurotrauma Section, Colombian Association of Neurological Surgeons. E-mail: andresrubiano@aol.com. ${ }^{2}$ Professor of Research, South Colombian University, Neiva (Colombia), Director, D-Neuropsy Research Group, South Colombian University (Colombia), Research Associate, MEDITECH Foundation, Neiva (Colombia). E-mail: neuro_angelamagnolia@hotmail.com. 